\title{
A dysflagellar mutant of Leishmania (Viannia) braziliensis isolated from a cutaneous leishmaniasis patient
}

Rogéria C Zauli', Jenicer KU Yokoyama-Yasunaka ${ }^{1}$, Danilo C Miguel ${ }^{1}$, Alexandre S Moura', Ledice IA Pereira², Ildefonso A da Silva Jr², Lucianna GN Lemes ${ }^{2}$, Miriam L Dorta ${ }^{2}$, Milton AP de Oliveira ${ }^{2}$, André N Pitaluga ${ }^{3}$, Edna AY Ishikawa ${ }^{4}$, Juliany CF Rodrigues ${ }^{5,6}$, Yara M Traub-Cseko ${ }^{3}$, A Tania Bijovsky ${ }^{1}$, Fátima Ribeiro-Dias ${ }^{2}$ and Silvia RB Uliana ${ }^{\text {** }}$

\begin{abstract}
Background: Parasites of the Leishmania genus alternate between the flagellated extracellular promastigote stage and intracellular amastigotes. Here we report the characterization of a Leishmania isolate, obtained from a cutaneous leishmaniasis patient, which presents peculiar morphological features.

Methods: The parasite was cultured in vitro and characterized morphologically using optical and electron microscopy. Identification was performed based on monoclonal antibodies and internal ribosomal spacer typing. In vitro macrophage cultures, murine experimental models and sand fly infections were used to evaluate infectivity in vitro and in vivo.

Results: The isolate was identified as Leishmania (Viannia) braziliensis. In the atypical promastigotes grown in culture, a short flagellum surrounded or interrupted by a protuberance of disorganized material was observed. A normal axoneme was present close to the basal body but without elongation much further outside the flagellar pocket. A disorganized swelling at the precocious end of the axoneme coincided with the lack of a paraflagellar rod structure. The isolate was able to infect macrophages in vitro, induce lesions in BALB/C mice and infect Lutzomyia longipalpis.

Conclusions: Notwithstanding the lack of an extracellular flagellum, this isolate infects macrophages in vitro and produces lesions when inoculated into mice. Moreover, it is able to colonize phlebotomine sand flies. Considering the importance attributed to the flagellum in the successful infection and survival of Leishmania in the insect midgut and in the invasion of macrophages, these findings may bring new light into the infectious mechanisms of L. (V.) braziliensis.
\end{abstract}

Keywords: flagellum, mutant, Leishmania, electron microscopy.

\section{Background}

Leishmania spp. are the etiological agents of a group of diseases known as leishmaniasis. The complex clinical presentations vary from localized cutaneous lesions to fatal visceral involvement. Leishmaniasis is endemic in the whole tropical and subtropical world with estimates of 12 million people currently infected. This protozoan

\footnotetext{
* Correspondence: srbulian@icb.usp.br

'Departamento de Parasitologia, Instituto de Ciências Biomédicas,

Universidade de São Paulo, São Paulo, 05508-900, Brasil

Full list of author information is available at the end of the article
}

parasite undergoes a complex life cycle alternating between the phlebotomine sand fly vector and a mammalian host. The vector is infected by the ingestion of a contaminated bloodmeal containing amastigotes. In the insect midgut, parasites transform into promastigotes, spindle-shaped, highly motile forms with a single flagellum that emerges from the anterior flagellar pocket. Inside the insect's gut, Leishmania parasites differentiate into several distinct developmental stages until they finally transform into metacyclic promastigotes. Each of these stages is characterized by morphological and

\section{Biomed Central}

(c) 2012 Zauli et al; licensee BioMed Central Ltd. This is an Open Access article distributed under the terms of the Creative Commons Attribution License (http://creativecommons.org/licenses/by/2.0), which permits unrestricted use, distribution, and reproduction in any medium, provided the original work is properly cited. 
functional changes that warrant their survival in the fly. After blood digestion and degradation of the peritrophic membrane the parasites attach to the gut epithelium where they multiply. In the case of Old World sand flies and parasites the attachment is mainly due to interactions provided by the major promastigote surface molecule, the lipophosphoglycan (reviewed in [1]). The mechanism is not so clear in New World vector-parasite pairs, but might involve lectins (reviewed in [2]). The differentiation into infective metacyclic promastigotes coincides with the detachment from the gut and migration towards the stomodeal valve [1]. Flagellar length varies from 10 to 20 micrometers in promastigotes [3]. In addition to conferring motility to the promastigote, the flagellum has been shown to be involved in the attachment to the gut in the female phlebotomine sand fly and to environmental sensing [3-6]. In some instances, the lack of flagellum was associated with failure to survive in the phlebotomine fly [7].

Transmission to the vertebrate host, including wild reservoirs, domestic animals and humans, occurs during the insect bite by the inoculation of metacyclic promastigotes into the skin. These are engulfed by mononuclear phagocytic cells and transform into amastigotes, round-shaped forms lacking an external flagellum. The invasion of macrophages by Leishmania (L.) donovani has also been related to flagellar attachment [8].

L. (V.) braziliensis is the most common Leishmania species in the Americas and the most important causative agent of cutaneous and mucocutaneous leishmaniasis in Brazil [9]. In this paper, we describe a morphologically atypical Leishmania isolate, obtained from a cutaneous leishmaniasis patient in Brazil.

\section{Methods}

\section{Parasites}

The MHOM/BR/2006/EFSF isolate, referred herein as EFSF6, was obtained from a patient attending the Anuar Auad Tropical Diseases Hospital in the city of Goiânia, Goiás, Brazil. The patient was infected in Minaçu, a municipality of Goiás State and presented five ulcerated lesions distributed in both arms. The lesions had appeared three months before the diagnosis. The patient was cured after treatment with a 20-day course of pentavalent antimonial. As part of the diagnostic procedure, skin biopsies were taken and the tissue was homogenised and inoculated into Grace's medium (SigmaAldrich Chem. Co., St Louis, MO, USA) supplemented with $20 \%$ fetal calf serum (FCS) [10].

The isolate was typed by PCR of ribosomal DNA, glucose-6-phosphate dehydrogenase and META2 gene as described [11-13] and identified as L. (V.) braziliensis. Soon after isolation, the culture was frozen and further tests were performed with freshly recovered cultures with a low passage number (third to seventh).

Atypical promastigotes of the EFSF6 isolate or typical forms of the L. (V.) braziliensis MHOM/BR/75/M2903 reference strain were grown in M199 liquid medium (Sigma-Aldrich) supplemented with $10 \%$ fetal calf serum (FCS; Invitrogen) and 2\% sterile male human urine, or in Grace's medium supplemented with $20 \%$ FCS and 2 $\mathrm{mM}$ L-glutamine (Sigma) and incubated at $22-26^{\circ} \mathrm{C}$.

Analysis of the parasite's morphology by optical microscopy was performed by applying and spreading slightly $10 \mu \mathrm{l}$ of culture onto a slide. The material was left to dry at room temperature and fixed and stained with the Instant Prov kit (Newprov, Pinhais-Paraná, Brazil).

\section{Monoclonal antibody typing}

For slide preparation, promastigotes were centrifuged at $1000 \times g$ for $10 \mathrm{~min}$, washed once in PBS pH $7.2(2.5$ $\mathrm{mM} \mathrm{NaH} \mathrm{PO}_{4}, 7.4 \mathrm{mM} \mathrm{Na} \mathrm{HPO}_{4}, 137 \mathrm{mM} \mathrm{NaCl}$ ), applied to the slides, dried and fixed with acetone for 15 $\min$.

The following monoclonal antibodies were used: B2, B5, B11, B12, B18, B19, M2, T3, CO2, L1, WIC, W1, W2, N2, N3 and WA2, according to methods described previously [14-16]. The $\mathrm{B}$ and $\mathrm{N}$ series react with species of the subgenus L. (Viannia); M2, T3, WIC.79.3, W1, W2 and WA2 react with parasites of the subgenus L. (Leishmania). CO2, and L1 are group-specific and react with members of subgenera Viannia and Leishmania, Endotrypanum and some species of the genus Trypanosoma. The monoclonal B18 is specific for $L$. (V.) braziliensis.

\section{Ribosomal Internal Transcribed Spacer (ITS) amplification and cloning}

Genomic DNA was obtained from cultured parasites as described previously [17]. Amplification of the ribosomal DNA internal transcribed spacers 1 and 2 (ITS1, ITS2) and $5.8 \mathrm{~S}$ ribosomal DNA (5.8S) (approximately $1 \mathrm{~kb}$ ) was performed with primers IR1 (5' GTA GGT GAA CCT GCA GCA GCT GGA TCA TT 3') and IR2 (5' GCG GGT AGT CCT GCC AAA CAC TCA GGT CTG 3') or 5.8R (5'GGA AGC CAA GTC ATC CAT C $\left.3^{\prime}\right)$ as described [18]. Amplified products were cloned into TOPO TA ${ }^{\circledR}$ (Invitrogen). GenBank accession numbers for sequences determined or used in this study are listed in the legend of Additional File 1.

Nucleotide sequences were analyzed using the program BioEdit http://www.mbio.ncsu.edu/BioEdit/bioedit. html and aligned with sequences from GeneBank using ClustalW [19]. The resulting alignments were manually refined. Parsimony and bootstrap analyses were carried out using PAUP* 4.0 [20] with 100 replicates of a 
random addition sequence followed by branch swapping (RAS-TBR), as previously described [21].

\section{Infection of macrophages in vitro}

Bone marrow-derived macrophages (BMDM) were obtained from BALB/c mice as described by [22]. BMDM were counted and distributed in 24-well plates on round coverslips $\left(3 \times 10^{5}\right.$ macrophages per well $)$ containing RPMI 1640 medium with 10\% FCS and 5\% L929 cell conditioned medium and allowed to adhere overnight at $37^{\circ} \mathrm{C}$, at $5 \% \mathrm{CO}_{2}$. Infections of macrophages were performed using ratios of 15 stationary-phase promastigotes per macrophage. Infected macrophage cultures were kept at $33^{\circ} \mathrm{C}, 5 \% \mathrm{CO}_{2}$ for $3 \mathrm{~h}$ in RPMI 1640 medium with $10 \%$ FCS and then washed twice with sterile PBS to remove free promastigotes. After $48 \mathrm{~h}$ of incubation, slides were fixed in methanol and stained with the Instant Prov kit (Newprov, Pinhais-Paraná, Brazil) for subsequent examination under light microscopy.

\section{Infection of mice}

Female BALB/c mice $(n=5-7)$ were infected in the left ear by intradermal inoculation of $1 \times 10^{5}$ stationaryphase promastigotes grown in M199 liquid medium. Stationary-phase promastigotes $\left(5 \times 10^{6} / 50 \mu \mathrm{L}\right.$ of PBS $)$ grown in Grace's medium were injected into the hind left footpads of C57BL/6 and BALB/c mice $(n=6)$. During 12 weeks, the evolution of lesions was evaluated by measuring the thickness of the infected ear or footpad using a calliper.

\section{Electron microscopy}

Logarithmic-phase promastigotes $\left(5 \times 10^{7} / \mathrm{mL}\right)$, washed twice with PBS pH 7.2, or tissue fragments, were fixed in a solution containing $2.5 \%$ glutaraldehyde in $0.1 \mathrm{M}$ cacodylate buffer, $\mathrm{pH} 7.2$, for $24 \mathrm{~h}$ at $4^{\circ} \mathrm{C}$. After fixation, the material was washed twice in $0.1 \mathrm{M}$ sodium cacodylate buffer, $\mathrm{pH} 7.2$ and post-fixed for $30 \mathrm{~min}$ in a solution containing $1 \% \mathrm{OsO}_{4}, 1.25 \%$ potassium ferrocyanide and $5 \mathrm{mM} \mathrm{CaCl}_{2}$ in $0.1 \mathrm{M}$ cacodylate buffer, washed in the same buffer, dehydrated in acetone series, and embedded in Epon or Spurr resin. Ultrathin sections were obtained in a Leica Ultramicrotome, stained with uranyl acetate and lead citrate and observed under a Zeiss 900 or a JEOL 100 CX transmission electron microscope operating at $80 \mathrm{kV}$. Images were recorded with a MegaView III camera (Olympus Soft Imaging Solutions) using the ITem Software. For scanning electron microscopy, cells were fixed as before, adhered to poly-L-lysine-coated coverslips, post-fixed for $30 \mathrm{~min}$ in a solution containing $1 \% \mathrm{OsO}_{4}, 1.25 \%$ potassium ferrocyanide and $5 \mathrm{mM} \mathrm{CaCl}_{2}$ in $0.1 \mathrm{M}$ cacodylate buffer, washed in the same buffer, dehydrated in ethanol series, critical point dried, and coated with gold in a Balzers gold sputtering system. Cells were observed under a Jeol JSM 6340F field emission scanning electron microscope operating at $5 \mathrm{kV}$.

\section{Rearing of Sand flies and artificial infection}

L. longipalpis were captured at Gruta da Lapinha, Minas Gerais, Brazil, and maintained in an insectary at Instituto Oswaldo Cruz, FIOCRUZ. Capture, maintenance and colonization in laboratory conditions were performed according to Brazil and Brazil [23]. Insects were sugar fed on $30 \%$ sucrose solution ad libitum.

For artificial blood-feeding or infection with the EFSF6 isolate or typical forms of the L. (V.) braziliensis MHOM/BR/75/M2903, three-day-old female sand flies were fed through chick skin membrane on hamster blood containing $10^{7}$ promastigotes $/ \mathrm{ml}$.

\section{Quantification of infection}

DNA was extracted from individual insects collected at 24, 72 or 96 hours post artificial infection with $L$. (V.) braziliensis MHOM/BR/75/M2903 or EFSF6 using wizard SV Genomic kit (Promega). Parasite load was assessed as described before [24]. qPCR reactions were performed using the SYBR Green PCR Master Mix (Applied Biosystems) and kDNA [25] and 18S primers [24]. Standard curves were constructed using serial dilutions of L. braziliensis and L. longipalpis DNA. The relative quantification was calculated using the $2^{-\Delta \Delta C t}$ method [26]. The Ct values of the $18 \mathrm{~S}$ reference gene from $L$. longipalpis were used for data normalization.

\section{Ethical aspects}

This study was approved by the Ethics Committee in Human and Animal Research of the HC/UFG and a consent letter was signed by the patient. All animal procedures were approved by the FIOCRUZ bioethics committee (CEUA - protocol number P0-116-02) and by ICB-USP Ethics Committee on Animal Experimentation.

\section{Statistical analysis}

Results are presented as media \pm SEM and data were analyzed by GraphPad Prism 4.0 Software (San Diego, CA, USA), using the Kruskal-Wallis and Mann-Whitney tests $(\mathrm{p}<0.05)$.

\section{Results}

Isolation of EFSF6 parasites and preliminary morphological characterization

EFSF6 parasites were initially obtained from skin biopsy homogenates cultured in Grace's medium. The observation of EFSF6 parasites recovered in primary in vitro cultures revealed an unusual morphology, distinct from conventional promastigotes: under phasecontrast, parasites looked round and were deposited at 
the bottom of the flasks (Figure 1A), while usual promastigotes appear as motile forms sometimes grouped in rosettes, as exemplified by the $L$. (V.) braziliensis reference strain M2903 (Figure 1B). Motility of EFSF6 parasites was greatly diminished as compared to M2903 cultures. While flagellated promastigotes exhibited a continuous oscillation pattern accompanied by rapid dislocation across the field of some parasites, EFSF6 cells only exhibited a fine vibrating pattern, without any significant displacements (Additional Files 2 and 3).

Optical microscopy revealed that most EFSF6 cells were round and presented a small and round protrusion at the body surface in the anterior region (Figure 1C), which was reminiscent of the rudimentary flagellum observed in the amastigotes. About 2-3\% of EFSF6 parasites observed under optical microscopy presented a visible short flagellum, extending from the anterior end of the cell while all M2903 promastigotes were flagellated cells (Figure 1D).

The growth pattern of EFSF6 promastigotes was analysed using two different culture media. Both in Grace's and M199 the EFSF6 parasites were able to multiply and reach stationary-phase at a similar rate and to the same density as the control L. (V.) braziliensis M2903 strain (Figure 2A and 2B). The morphology of EFSF6 stationary-phase parasites remained unaltered when compared to that observed for logarithmic-phase promastigotes (Figure 2C, D).

We also tested whether promastigote morphology varied with temperature. Varying the incubation temperature of cultures from 22 to $26^{\circ} \mathrm{C}$ did not induce any changes in morphology and weekly subcultures at these temperatures for 2 months showed that the morphological phenotype was a stable characteristic (data not shown).
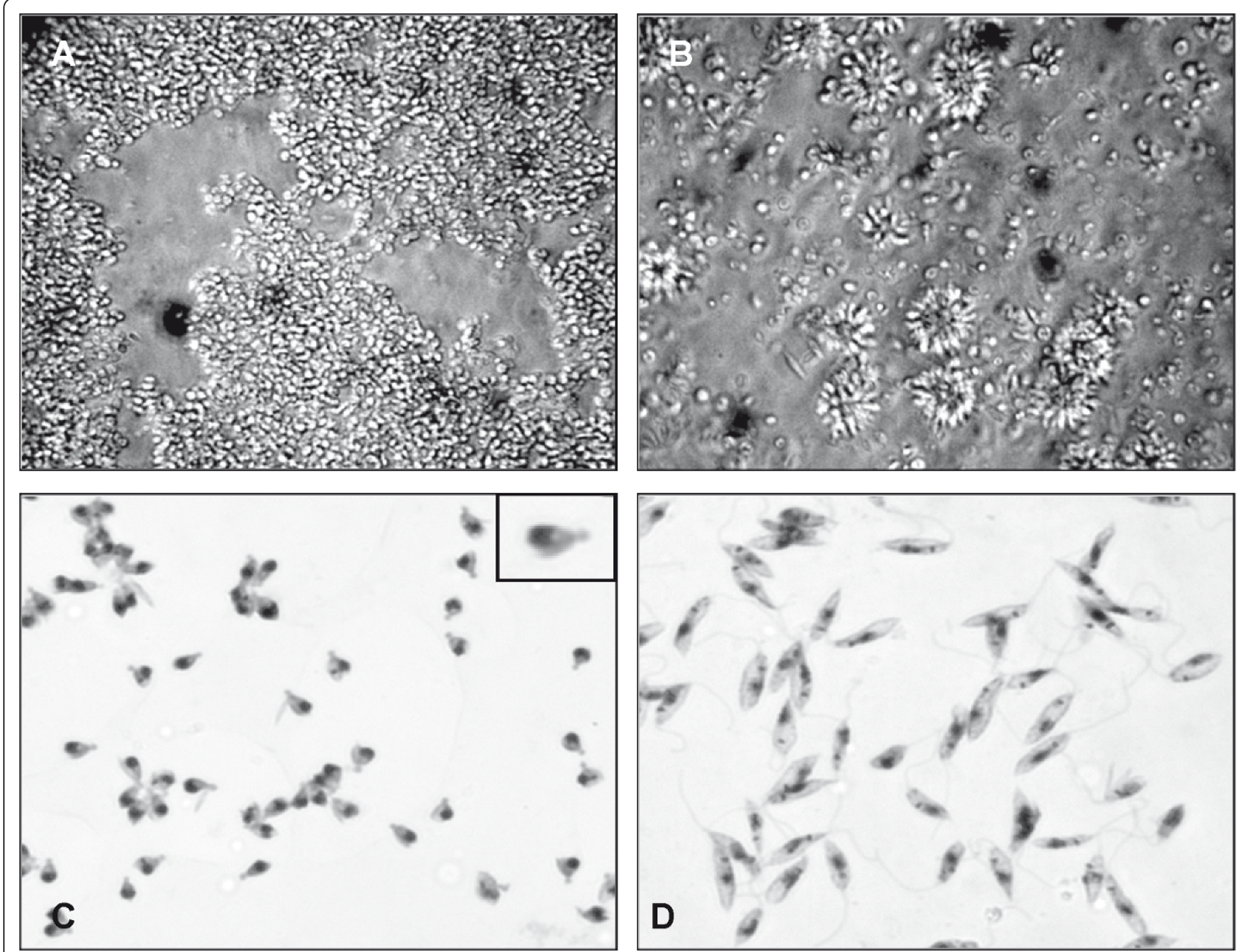

Figure 1 Morphology of the EFSF6 isolate. Phase contrast image (A, B) of EFSF6 (A) and M2903 (B) live promastigote cultures; magnification 400x. Microphotographs of stained EFSF6 (C) and M2903 (D) promastigotes; magnification 1,000x. Box in (C) shows the atypical flagellum (arrow). 

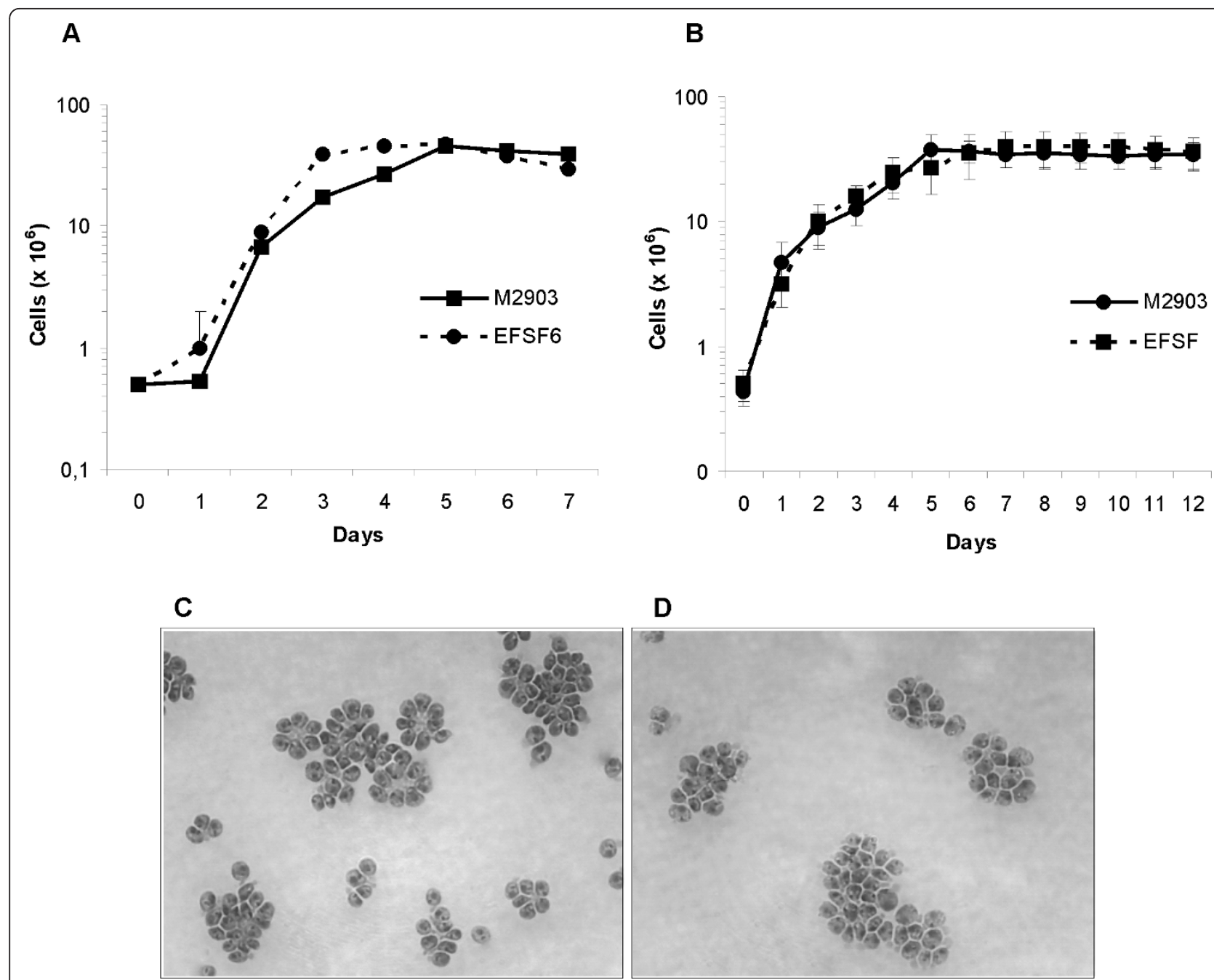

D

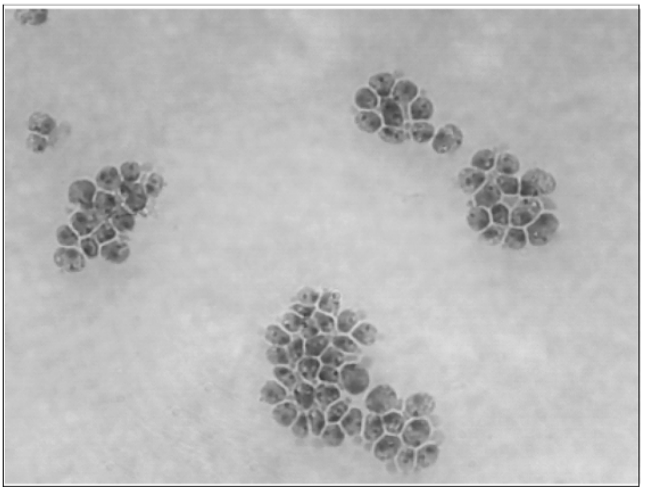

Figure 2 Growth curves of EFSF6 and M2903. Promastigotes cultivated in medium 199 with 10\% FCS and 2\% sterile human male urine (A) or in Grace's media with 20\% FCS (B). Logarithmic-phase (C) and stationary-phase (D) EFSF6 promastigotes in Grace's media; magnification 1000x.

\section{Identification of the EFSF6 isolate}

Although the isolate was obtained from a patient with localized skin lesions and had been previously identified as L. (V.) braziliensis [13], the unusual morphology urged a more thorough identification assessment. In vitro cultured parasites were tested using a panel of 16 monoclonal antibodies described as tools for Leishmania identification and typing [14-16]. Positive reactions were detected with monoclonal antibodies L1, CO2, B12, B5 and B18, confirming the identification of a parasite belonging to the Leishmania genus and typing the isolate as L. (V.) braziliensis. Reactions with monoclonal N2, usually positive against the reference strain M2903, were negative with isolate EFSF6 (data not shown).

Molecular confirmation was also based on sequencing of the ribosomal internal transcribed spacers 1 and 2 and 5.8S rDNA. These molecular markers are widely used in the identification of trypanosomatids and can be employed to determine phylogenetic relationships and also assist in the identification of a particular isolate. A 985 nt ITS1/5.8S/ITS2 sequence was determined for the EFSF6 isolate. Sequence comparison over the conserved $5.8 \mathrm{~S}$ sequence showed $100 \%$ identity with all other Leishmania sequences, as expected. Alignment of ITS1 and 2 segments indicated $97-98 \%$ identity with $L$. (V.) braziliensis and $L$. (V.) guyanensis sequences (data not shown). A maximum parsimony analysis grouped the EFSF6 isolate with L. (V.) braziliensis (Additional File 1).

\section{Detailed morphological characterization of $L$. (V.) braziliensis EFSF6 parasites}

Observation of EFSF6 promastigotes under scanning electron microscopy confirmed the fusiform or pyriform morphology of the cell bodies for most promastigotes (Figure 3A). The presence of longitudinal grooves, often twisted along the body of the parasite was noted. Over 


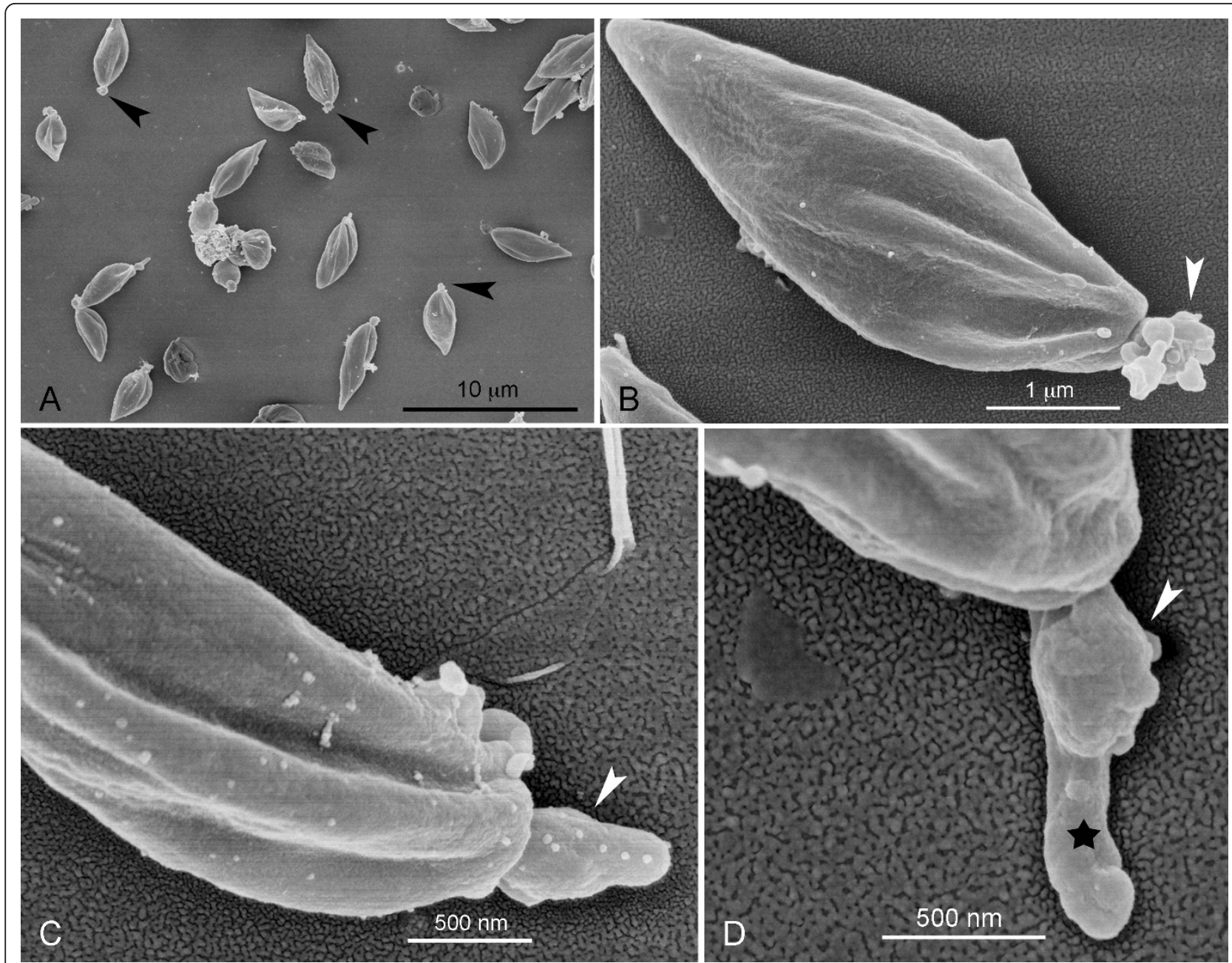

Figure 3 Scanning electron microscopy of EFSF6 promastigotes. (A) logarithmic phase promastigotes with atypical flagellum (arrow). (B) and (C) presence of longitudinal grooves and rounded micellar corpuscles (arrow). (D) anterior region (flagellum) of promastigotes.

$85 \%$ of the logarithmic phase EFSF6 promastigotes showed the atypical flagellum surrounded or interrupted by a protuberance of disorganized material (Figure 3B$\mathrm{D}$, arrowheads). In addition, some parasites also presented one small flagellum externalised from the flagellar pocket that appeared associated with a disorganised structure (Figure 3D, star). Many parasites had vesicles adhered to the cell surface (Figure 3C).

EFSF6 parasites along with the reference strain were also examined using transmission electron microscopy of fixed promastigotes. The M2903 promastigotes showed a typical flagellar structure formed by the axoneme and paraflagellar rod (Figure 4A-C, arrowhead). In a longitudinal section of M2903 promastigotes, it was possible to observe a normal kinetoplast present in the anterior region, the flagellar pocket, some acidocalcisomes and a nucleus. EFSF6 cells showed a typical single mitochondria containing the distinctive array of kinetoplast DNA (Figure 4D-E). The flagellar basal body with an erupting flagellum with normal structure was observed (Figure 4D-G). At the anterior extremity and where the flagellum is due to emerge from the flagellar pocket, a disorganized bulge (indicated by the arrowhead) was noted (Figure 4D-G). This disorganized swelling coincides with the position where the paraflagellar rod should be inserted. From that point towards the exterior of the cell, a disassembly of the axoneme was observed. In high magnification, it was also possible to detect a disorganization of the membrane that surrounds the abnormal flagellar structure (Figure 4F-G, arrow). In some cells the axoneme leaving the flagellar pocket showed a normal organization (Figure 4G, star), with features similar to those observed by scanning electron microscopy (Figure 3D, star). Acidocalcisomes (Ac) and lipid inclusions (stars) were also observed in the EFSF6 cells (Figure 4D-E). 


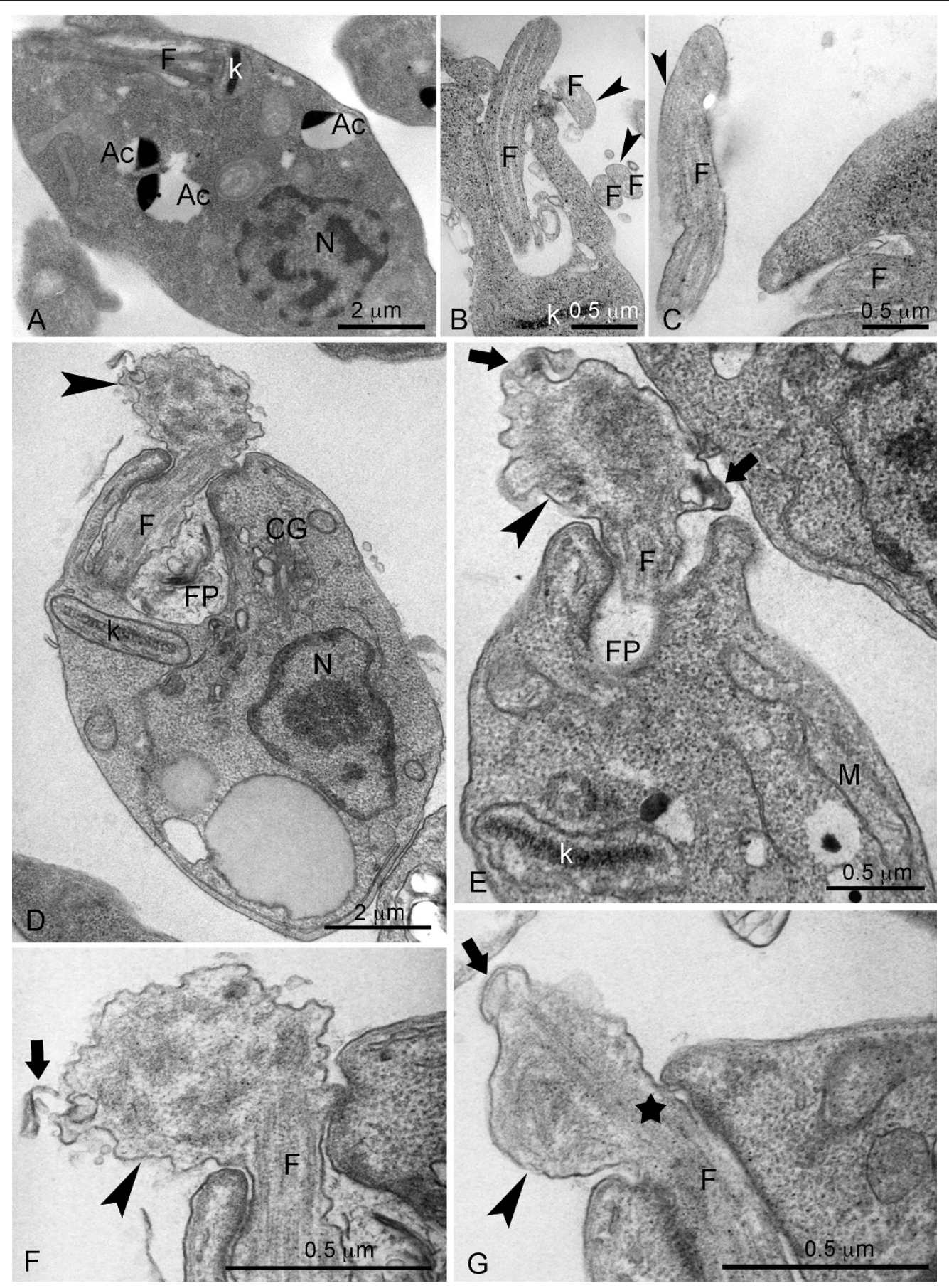

Figure 4 Ultrathin section of promastigotes of the reference strain M2903 (A-C) and EFSF6 parasites (D-G). These images illustrate the ultrastructure of promastigotes from $3^{\text {rd }}$ day of culture analysed by transmission electron microscopy. (N) nucleus, (K) kinetoplast, (FP) flagellar pocket, (F) flagellum and (Ac) acidocalcissomes.

\section{In vitro and in vivo infectivity of the EFSF6 isolate}

To test whether the parasites recovered were infective, we performed in vitro and in vivo infection experiments. The typical pattern of macrophage infection for $L$. $(V$.) braziliensis consists of small vacuoles with few amastigotes per vacuole (Figure 5A). EFSF6 promastigotes from in vitro culture were able to infect murine bone marrow derived macrophages, which became heavily parasitized (Figure 5B) with intracellular amastigotes distributed in tight vacuoles. 

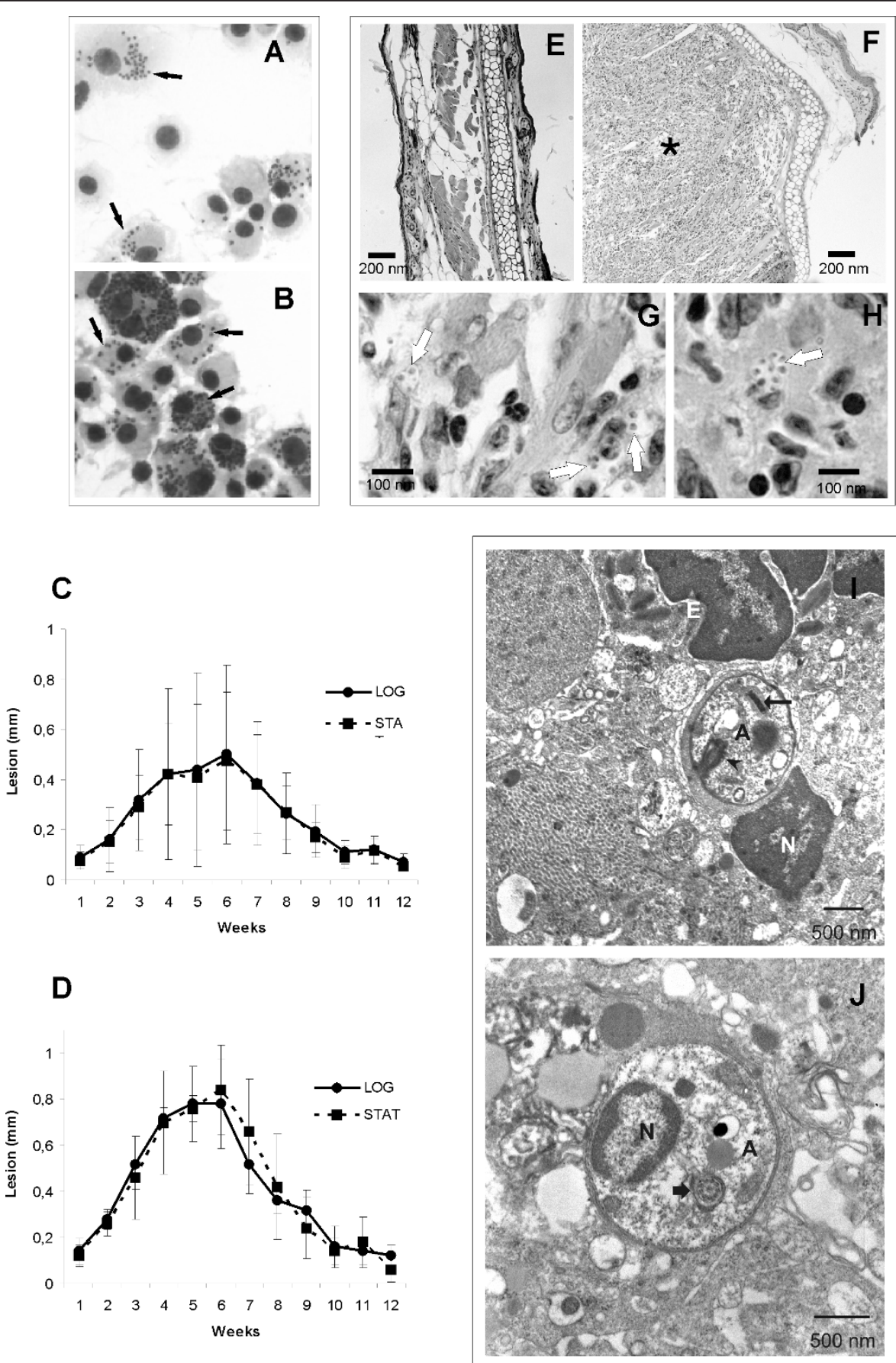

Figure 5 Infectivity of EFSF6 promastigotes in vitro and in vivo. (A-B) Susceptibility of BMDM to infection with the EFSF6 isolate. Macrophages were infected with stationary-phase promastigotes of $L$. (V.) braziliensis reference strain (MHOM/BR/75/M2903) (A) or with the EFSF6 isolate (B) using a ratio of 15 parasites/macrophage for 3 hours at $33^{\circ} \mathrm{C}$ and $5 \% \mathrm{CO}_{2}$. The infection was maintained for 6 days under these conditions (magnification 1000x). (C-J) Infectivity of EFSF6 promastigotes to mice. (C-D) Lesion progression after inoculation of $5 \times 10^{6}$ EFSF6 promastigotes in the hind footpad of BALB/C (C) or C57BL/6 (D) mice. Parasites were from the $2^{\text {nd }}$ (solid line) or $6^{\text {th }}$ day of culture (dashed line). Results shown are the mean and standard deviation of lesion size in groups of 6 mice. Lesion size is the difference between the thickness of infected and non-infected contralateral footpads. (E-H) Histopathological analysis of the uninfected (E) or EFSF6-infected (F-H) ear of a BALB/C mouse. Tissue was fixed 3 weeks after the inoculation of $1 \times 10^{5}$ EFSF6 promastigotes. Magnification: 40× (E-F) and 1000× (G-H). (I-J)

Transmission electron microscopy showing ear fragments from mice infected with EFSF6. Arrows point to amastigotes surrounded by tight parasitophorous vacuoles; the thick arrow indicates a kinetoplast and the thin arrow shows the amastigote rudimentary flagellum. (A) amastigote, (N) nucleus, (E) eosinophil. 
EFSF6 promastigotes were also used to infect BALB/c and $\mathrm{C} 57 \mathrm{Bl} / 6$ mice. The injection of $5 \times 10^{6}$ parasites at the hind footpad of either mice lineage induced small lesions, apparent from week 3 and reaching their peak at week 5 or 6 (Figure 5C, D). By week 10 footpads showed no macroscopic signs of infection. Lesion progression was similar, irrespective of the day of culture of EFSF6 promastigotes inoculated (Figure 5C, D). Parasites isolated from mouse footpad lesions and cultured in Grace's medium maintained the same morphological characteristics of those parasites isolated directly from the patient fragment biopsy (data not shown).

$\mathrm{BALB} / \mathrm{c}$ mice were also infected at the ear dermis. Swelling at the inoculation site was noted by week 3, peaked at week 6 and, by week 10, all animals were clinically cured (data not shown). Histopathological sections of the ear from infected mice showed a mononuclear inflammatory reaction and the presence of parasitized macrophages (Figure 5F-H). Biopsies of BALB/c ears infected with EFSF6 were also analysed by transmission electron microscopy. The examination of these tissue biopsies allowed the identification of typical amastigotes inside host cells, confirming the infectivity of the EFSF6 promastigotes. These parasites were contained inside tight vacuoles and exhibited the characteristic kDNA structure, being therefore indistinguishable from typical Leishmania amastigotes (Figure 5I-J).

\section{Infectivity of EFSF6 parasites to phlebotomine sand flies}

Lastly, we investigated whether phlebotomine sand flies could be successfully infected with EFSF6 parasites. To that purpose, female L. longipalpis were fed on hamster blood containing EFSF6 or M2903 L. braziliensis promastigotes. After various time intervals, DNA was prepared from individual flies and parasites were quantified by qPCR. Flies fed with the EFSF6 isolate were successfully infected, as shown by the presence of amplification 72 and 96 hours post-feeding (Figure 6). All flies analysed after 96 hours had already expelled the remains of the blood meal. There was no difference in the number of infected flies 24, 72 or 96 hours post-feeding EFSF6 or M2903 parasites. A small but not significant difference was observed at $48 \mathrm{~h}$ post-feeding. In particular, a comparable number of positive flies after 96 hours, whether infected with EFSF6 or M2903 parasites, indicated that both parasites survived proteolytic blood digestion and excretion of blood and peritrophic membrane remnants, being able to establish a successful infection in the insect vector.

\section{Discussion}

In this paper we describe the morphological and biological characteristics of a parasite isolated from a cutaneous leishmaniasis patient. The morphology of this

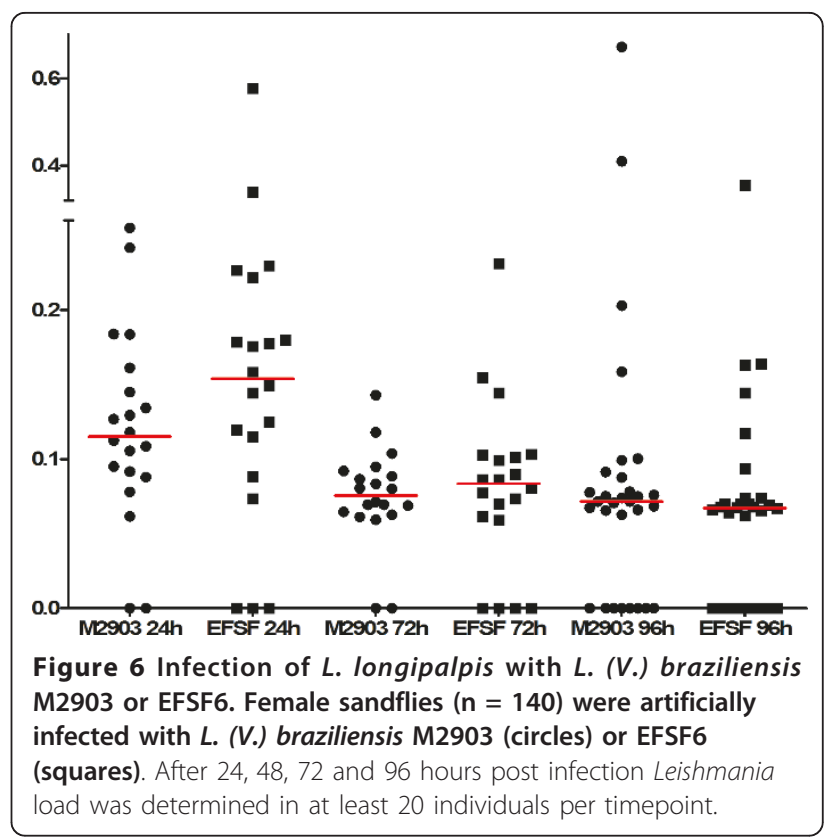

isolate was atypical ever since it was recovered in vitro from the tissue biopsy. Initially, the observation of the cultures raised doubts even as to whether it was indeed a Leishmania isolate. Identification methods using several different criteria, including monoclonal antibodies and genotyping by ribosomal RNA conserved and variable sequences led to a definite typing as $L$. (V.) braziliensis. To our knowledge, the morphological characteristics exhibited by EFSF6 parasites in culture medium that imitates the insect gut have not been previously described on any field isolate. Strictly speaking these parasites could not be called promastigotes since they do not exhibit a free anterior flagellum. In the course of this paper, however, we took the license to call them atypical promastigotes. They are able to grow in in vitro cultures and the phenotype is stable.

A morphological analysis by optical and electron microscopy suggested that these parasites exhibit a defective machinery for flagellum elongation. The basal body and initial portion of the axoneme are present but the axoneme does not elongate further after the exit from the flagellar pocket. The absence of the paraflagellar rod, characteristic of trypanosomatids is striking. At the emergence of this short axoneme, a deposit of material is found, suggesting that proteins synthesized to travel along the flagellum are trapped at this position.

The causes for this mutated phenotype are presently unknown. The paraflagellar rod contains 2 proteins, PFR1 and PFR2. The functional role of the paraflagellar rod in $L$. (L.) mexicana was evaluated using mutants lacking the PFR1 and PFR2 genes. Ablation of PFR1 and PFR2 proteins in $L$. (L.) mexicana promastigotes induced 
severe perturbations in flagellar wave propagation; however, the length of the axoneme was not changed in these null mutants $[27,28]$. In these paraflagellar rod null mutants, transmission electron microscopy revealed the presence of a disorganized material connected to the axoneme through thin filaments, indicating that these two proteins are essential for the assembly of the paraflagellar rod structure.

Several studies have revealed the presence of different proteins probably involved in the assembling of the flagellar structure in Leishmania. In $L$. (L.) donovani, the lack of a gene that encodes the $70 \mathrm{kDa}$ subunit of the outer dynein arm docking complex exhibited shorter and rounded cell bodies and a pronounced shortening of the flagellar length with severe alterations in motility and a strongly reduced cellular growth. However, these parasites have a typical paraflagellar rod structure [29]. Ablation of genes encoding the dynein-2 heavy chain [30], ADF/cofilin [31], or MAP kinase proteins [32] in $L$. (L.) mexicana resulted in perturbation of cell motility, lack of the paraflagellar rod and disorganization of the axoneme. All these features are similar to those observed in the $L$. $(V$.) braziliensis EFSF6 promastigotes, which underlines the complexity of the machinery responsible for assembling the flagellar and paraflagellar structures. Other proteins, such as a thymidine kinase and mitogen-activated protein kinase have been implicated in normal elongation of the flagellum and in this case the lack of the protein results in a lengthened flagellum [33,34].

The flagellum of promastigotes has been implicated in macrophage invasion by promastigotes in $L$. (L.) donovani [8]. In spite of the striking morphological differences, promastigotes of the EFSF6 isolate were infective in vitro to BMDM as well as to peritoneal macrophages (data not shown). Furthermore, inoculation of EFSF6 promastigotes into $\mathrm{C} 57 \mathrm{Bl} / 6$ or $\mathrm{BALB} / \mathrm{c}$ mice gave rise to disease patterns indistinguishable from other $L$. (V.) braziliensis strains, which induce a self-healing disease in these experimental models. However, the question of whether this isolate was transmitted to the patient as such is elusive.

On the other hand, a relationship between an intact flagellum and survival in the insect vector has been observed [6] (and references therein). An intact flagellum is obviously important for motility, which should serve a function in the necessary migrations the parasite has to perform while inside the fly. Furthermore, the attachment via flagellum to the epithelial surface seems to be important. In fact, an aflagellated $L$. (L.) amazonensis mutant has been shown to be unable to infect $L$. longipalpis [7].

$L$. longipalpis is not the natural vector of $L$. (V.) braziliensis, but it is a permissive vector, supporting the growth of several Leishmania species, including $L$. $(V$.) braziliensis. It has been suggested that in specific Old World vectors Leishmania surface lipophosphoglycan is determinant to successful attachment to the gut, while in permissive vectors other factors, including the interaction with the flagellum gain importance [35]. The natural infection of sand flies occurs by the ingestion of amastigote-containing blood but they can be infected with promastigotes [36]. The infection of L. longipalpis with the EFSF6 isolate showed that these atypical promastigotes are perfectly able to survive in the fly for the first 96 hours, or, in other words, after the expulsion of the digested blood and degraded peritrophic membrane. It will also be of utmost interest to find out whether these parasites can be transmitted by the bite of the phlebotomine sand fly, which could give us some indication about the likelihood of finding this type of mutated parasites in the field.

The EFSF6 isolate opens several possibilities to a new understanding of the roles of the flagellum in the life cycle, transmission and pathogenesis. We still do not know what is missing in this mutant and several approaches are being considered to investigate that. Meanwhile, we believe this isolate demonstrates that the variability and infectious properties of Leishmania in the field have not been completely understood and uncovered.

\section{Conclusions}

We have characterized a clinical isolate of $L$. (V.) braziliensis that presents as a natural dysflagellar mutant, where the axoneme is present but unable to elongate to constitute an external flagellum. In spite of that, these parasites are able to infect macrophages in vitro, cause disease in mice and infect phlebotomine sand flies. This isolate may be able to provide valuable information about the infectious processes used by the parasite both in the vertebrate and invertebrate hosts.

\section{Additional material}

\footnotetext{
Additional file 1: Molecular identification and phylogenetic

relationships of the EFSF6 isolate. Dendrogram based on ITS1/5.8S/

ITS2 sequences from the following organisms: L. (L.) braziliensis EFSF6 [GenBank JQ061322], L. (L.) chagasi [GenBank AJ000305.1], L. (L.) donovani [GenBank AJ000293.1, AM901450.1], L. (L.) major [GenBank AJ000310.1, DQ300195], L. (L.) amazonensis [GenBank AJ000314], L. (L.) mexicana [GenBank AF466383, AF466380.1], L. (V.) guyanensis [GenBank FJ753387, AJ000299.1, AJ000300.1], L. (V.) braziliensis [GenBank AJ300483, AJ300484, AJ300483] e Leptomonas mirabilis [GenBank AY180153.1]. The numbers at the branches refer to parsimony percentage bootstrap values derived from 100 replicates.

Additional file 2: Phase contrast imaging of $L$. (V.) braziliensis M2903 promastigotes in M199 medium. Representative 30 seconds video, taken at $72 \mathrm{~h}$ of culture started with an inoculum of $1 \times 10^{6}$ parasites $/ \mathrm{ml}$. This movie shows the general movement of typical $L$. (V.)
} 
braziliensis promastigotes. Note the fast moving parasites that trave across the field and the typical rosettes.

Additional file 3: Phase contrast imaging of $L$. (V.) braziliensis EFSF6 promastigotes in M199 medium. Representative 30 seconds video, taken at $72 \mathrm{~h}$ of culture started with an inoculum of $1 \times 10^{6}$ parasites/ $\mathrm{ml}$. This movie shows that these parasites tend to gather in groups. No fast movement is observed. A vibration is noted on the clusters of cells.

\section{Acknowledgements}

We are thankful to Dr. Jeffrey J. Shaw for helpful discussions and to Raquel Senna Telhado and Rodrigo Jorge de Alcantara Guerra for help with the sand fly experiments. This work was supported by Conselho Nacional de Desenvolvimento Científico e Tecnológico, Fundação de Amparo à Pesquisa do Estado de São Paulo and CAPES (PROCAD/NF 179-2010). Fellowship support to RCZ was provided by CNPq and to DCM by FAPESP.

\section{Author details}

'Departamento de Parasitologia, Instituto de Ciências Biomédicas, Universidade de São Paulo, São Paulo, 05508-900, Brasil. ${ }^{2}$ Instituto de Patologia Tropical e Saúde Pública, Universidade Federal de Goiás, Goiânia, 74605-050, Brazil. ' ${ }^{3}$ Laboratório de Biologia Molecular de Parasitas e Vetores, Instituto Oswaldo Cruz, Fiocruz, Rio de Janeiro, 21045-900, Brazil. ${ }^{4}$ Núcleo de Medicina Tropical, Universidade Federal do Pará, Belém, 66055-240, Brazil. ${ }^{5}$ Laboratório de Ultraestrutura Celular Hertha Meyer, Instituto de Biofísica Carlos Chagas Filho, Universidade Federal do Rio de Janeiro, Rio de Janeiro, 21944-970, Brazil. ' Universidade Federal do Rio de Janeiro, Pólo Avançado de Xerém, Rio de Janeiro, Brazil.

\section{Authors' contributions}

ATB, MLD, FRD, SRBU conceived the study; RCZ, JKUYY, DCM, LIAP, IASJ, LGNL, MAPO, El worked in the isolation of the parasite, in vitro and in vivo characterization; RCZ, DCM, ASM, JCFR, ATB, worked on the morphological characterization of the parasite; ANP, YMTC performed the sand fly work; ATB, FRD, YTC and SRBU analyzed the data and wrote the manuscript. All authors read and approved the final version of the manuscript.

\section{Competing interests}

The authors declare that they have no competing interests.

Received: 16 December 2011 Accepted: 11 January 2012

Published: 11 January 2012

\section{References}

1. Kamhawi S: Phlebotomine sand flies and Leishmania parasites: friends or foes? Trends in Parasitology 2006, 22:439-445.

2. Ramalho-Ortigao JM, Saraiva EM, Traub-Csekö YM: Sand fly-Leishmania interactions: long relationships are not necessarily easy. Open Parasitol J 2010, 4:195-204.

3. Molyneux DH, Killick-Kendrick R: Morphology, ultrastructure and life cycles. In The Leishmaniases in Biology and Medicine. Edited by: Peters W, Killick-Kendrick R. London: Academic Press; 1987:1-119.

4. Killick-Kendrick R, Molyneux DH, Ashford RW: Leishmania in phlebotomid sandflies. I. Modifications of the flagellum associated with attachment to the mid-gut and oesophageal valve of the sandfly. Proc $R$ Soc Lond B Biol Sci 1974, 187:409-419.

5. Warburg A, Tesh RB, McMahon-Pratt D: Studies on the attachment of Leishmania flagella to sand fly midgut epithelium. J Protozool 1989, 36:613-617.

6. Bates PA: Leishmania sand fly interaction: progress and challenges. Curr Opin Microbiol 2008, 11:340-344.

7. Cuvillier A, Miranda JC, Ambit A, Barral A, Merlin G: Abortive infection of Lutzomyia longipalpis insect vectors by aflagellated LdARL-3A-Q70L overexpressing Leishmania amazonensis parasites. Cell Microbiol 2003, 5:717-728.

8. Forestier CL, Machu C, Loussert C, Pescher P, Spath GF: Imaging host cellLeishmania interaction dynamics implicates parasite motility, lysosome recruitment, and host cell wounding in the infection process. Cell Host Microbe 2011, 9:319-330.
9. Lainson R, Shaw JJ, Ward RD, Fraiha H: Leishmaniasis in Brazil. IX. Considerations on the Leishmania braziliensis complex. Importance of sandflies of the genus Psychodopygus (Mangabeira) in the transmission of L. braziliensis braziliensis in north Brazil. Trans R Soc Trop Med Hyg 1973, 67:184-196.

10. Oliveira MA, Pires Ada S, de Bastos RP, Lima GM, Pinto SA, Pereira LI, Pereira AJ, Abrahamsohn Ide A, Dorta ML, Ribeiro-Dias F: Leishmania spp. parasite isolation through inoculation of patient biopsy macerates in interferon gamma knockout mice. Rev Inst Med Trop Sao Paulo 2010, 52:83-88.

11. Uliana SR, Nelson K, Beverley SM, Camargo EP, Floeter-Winter LM: Discrimination amongst Leishmania by polymerase chain reaction and hybridization with small subunit ribosomal DNA derived oligonucleotides. J Eukaryot Microbiol 1994, 41:324-330.

12. Castilho TM, Shaw JJ, Floeter-Winter LM: New PCR assay using glucose-6phosphate dehydrogenase for identification of Leishmania species. J Clin Microbiol 2003, 41:540-546.

13. Zauli-Nascimento RC, Miguel DC, Yokoyama-Yasunaka JK, Pereira LI, Pelli de Oliveira MA, Ribeiro-Dias F, Dorta ML, Uliana SR: In vitro sensitivity of Leishmania (Viannia) braziliensis and Leishmania (Leishmania) amazonensis Brazilian isolates to meglumine antimoniate and amphotericin B. Trop Med Int Health 2010, 15:68-76.

14. McMahon-Pratt $D$, Bennett $E$, David JR: Monoclonal antibodies that distinguish subspecies of Leishmania braziliensis. J Immunol 1982, 129:926-927.

15. Shaw JJ, Ishikawa EA, Lainson R: A rapid and sensitive method for the identification of Leishmania with monoclonal antibodies using fluorescein-labelled avidin. Trans R Soc Trop Med Hyg 1989, 83:783-784.

16. Shaw JJ, De Faria DL, Basano SA, Corbett CE, Rodrigues CJ, Ishikawa EA, Camargo LM: The aetiological agents of American cutaneous leishmaniasis in the municipality of Monte Negro, Rondonia state, western Amazonia, Brazil. Ann Trop Med Parasitol 2007, 101:681-688.

17. Medina-Acosta E, Cross GAM: Rapid isolation of DNA from trypanosomatid protozoa using a simple mini-prep procedure. Mol Biochem Parasitol 1993, 59:327-329.

18. Cupolillo E, Grimaldi G Jr, Momen H, Beverley SM: Intergenic region typing (IRT): a rapid molecular approach to the characterization and evolution of Leishmania. Mol Biochem Parasitol 1995, 73:145-155.

19. Thompson JD, Higgins DG, Gibson TJ: CLUSTAL W: improving the sensitivity of progressive multiple sequence alignment through sequence weighting, position-specific gap penalties and weight matrix choice. Nucleic Acids Res 1994, 22:4673-4680

20. Swofford D, Paup L: Phylogenetic Analysis Using Parsimony (*and Other Methods). Version 4 Sunderland, Massachusetts: Sinauer Associates; 1998.

21. Ferreira RC, De Souza AA, Freitas RA, Campaner M, Takata CS, Barrett TV, Shaw JJ, Teixeira MM: A phylogenetic lineage of closely related trypanosomes (Trypanosomatidae, Kinetoplastida) of anurans and sand flies (Psychodidae, Diptera) sharing the same ecotopes in brazilian amazonia. J Eukaryot Microbiol 2008, 55:427-435.

22. Zamboni DS, Rabinovitch M: Nitric oxide partially controls Coxiella burnetii phase II infection in mouse primary macrophages. Infection and Immunity 2003, 71:1225-1233.

23. Brazil RP, Brazil BG: Biologia de Flebotomíneos neotropicais. In Flebotomíneos do Brasil. Edited by: Rangel EF, Lainson R. Rio de Janeiro: Editora Fiocruz; 2003:257-274.

24. Bezerra-Vasconcelos DR, Melo LM, Albuquerque ES, Luciano MC, Bevilaqua CM: Real-time PCR to assess the Leishmania load in Lutzomyia longipalpis sand flies: screening of target genes and assessment of quantitative methods. Exp Parasitol 2011, 129:234-239.

25. Degrave W, Fernandes O, Campbell D, Bozza M, Lopes U: Use of molecular probes and PCR for detection and typing of Leishmania - a mini-review. Mem Inst Oswaldo Cruz 1994, 89:463-469.

26. Livak KJ, Schmittgen TD: Analysis of relative gene expression data using real-time quantitative PCR and the 2(-Delta Delta C(T)) Method. Methods 2001, 25:402-408.

27. Santrich C, Moore L, Sherwin T, Bastin P, Brokaw C, Gull K, LeBowitz JH: A motility function for the paraflagellar rod of Leishmania parasites revealed by PFR-2 gene knockouts. Mol Biochem Parasitol 1997, 90:95-109.

28. Maga JA, LeBowitz JH: Unravelling the kinetoplastid paraflagellar rod. Trends in Cell Biology 1999, 9:409-413. 
29. Harder S, Thiel M, Clos J, Bruchhaus I: Characterization of a subunit of the outer dynein arm docking complex necessary for correct flagellar assembly in Leishmania donovani. PLoS Negl Trop Dis 2010, 4:e586.

30. Adhiambo C, Forney JD, Asai DJ, LeBowitz JH: The two cytoplasmic dynein-2 isoforms in Leishmania mexicana perform separate functions. Mol Biochem Parasitol 2005, 143:216-225.

31. Tammana TV, Sahasrabuddhe AA, Bajpai VK, Gupta CM: ADF/cofilin-driven actin dynamics in early events of Leishmania cell division. J Cell Sci 2010, 123:1894-1901

32. Erdmann M, Scholz A, Melzer IM, Schmetz C, Wiese M: Interacting protein kinases involved in the regulation of flagellar length. Mol Biol Cell 2006, 17:2035-2045.

33. Bengs F, Scholz A, Kuhn D, Wiese M: LmxMPK9, a mitogen-activated protein kinase homologue affects flagellar length in Leishmania mexicana. Mol Microbiol 2005, 55:1606-1615

34. Thiel M, Harder S, Wiese M, Kroemer M, Bruchhaus I: Involvement of a Leishmania thymidine kinase in flagellum formation, promastigote shape and growth as well as virulence. Mol Biochem Parasitol 2008, 158:152-162.

35. Myskova J, Svobodova M, Beverley SM, Volf P: A lipophosphoglycanindependent development of Leishmania in permissive sand flies. Microbes Infect 2007, 9:317-324.

36. Maia C, Seblova V, Sadlova J, Votypka J, Volf P: Experimental transmission of Leishmania infantum by two major vectors: a comparison between a viscerotropic and a dermotropic strain. PLOS Negl Trop Dis 2011, 5:e1181.

doi:10.1186/1756-3305-5-11

Cite this article as: Zauli et al:: A dysflagellar mutant of Leishmania (Viannia) braziliensis isolated from a cutaneous leishmaniasis patient. Parasites \& Vectors 2012 5:11.

\section{Submit your next manuscript to BioMed Central and take full advantage of:}

- Convenient online submission

- Thorough peer review

- No space constraints or color figure charges

- Immediate publication on acceptance

- Inclusion in PubMed, CAS, Scopus and Google Scholar

- Research which is freely available for redistribution

Submit your manuscript at www.biomedcentral.com/submit
Biomed Central 